\title{
Análisis en flujo permanente de los factores que inciden en la disminución de la capacidad hidráulica de la línea de conducción del sistema La Mica-Quito Sur
}

\author{
Andrade Ramos Juan Martin ${ }^{1}$, Escobar Cevallos Gilson Manuel², Paredes Méndez Diego Fernando ${ }^{3}$ \\ ${ }^{1}$ Ingeniero Civil, independiente, 2095286-0984699448,Av. Intervalles y Jacpi \\ mail: juanmartin.jma@hotmail.com \\ ${ }^{2}$ Ingeniero Civil, independiente, 022362097-0984963971, Av. Natalia Jarrín y Los Pinos \\ mail: gilson.escobar19@gmail.com \\ ${ }^{3}$ Ingeniero Civil, Master of Science in Water Science and Engineering, \\ Docente Facultad de Ingeniería, Ciencias Físicas y Matemática, \\ Jefe Unidad Diagnóstico Hidráulico EPMAPS, 0987509604, Quito-Ecuador \\ mail: dfparedes@uce.edu.ec
}

\begin{abstract}
Artículo científico enviado para su revisión el 20 de julio de 2018. Este trabajo fue posible gracias al apoyo de la Empresa Pública Metropolitana de Agua Potable y Saneamiento EPMAPS. Aceptado: septiembre 2018
\end{abstract}

\section{Resumen}

El sistema "La Mica - Quito Sur" dota de agua a la zona sur de Quito, en la actualidad transporta un caudal de 1560 1/s, menor a $1750 \mathrm{l} / \mathrm{s}$ para el cual fue diseñado, esta disminución se debe a factores que generan pérdidas de energía en la línea de conducción lo que disminuye su capacidad hidráulica. Para disponer de un modelo hidráulico actualizado, se realizó una intensa actividad. Se recopiló planos y bases de datos del sistema, información que fue validada in situ. A continuación se construyó, calibró y validó el modelo hidráulico, obteniendo de su análisis las características hidráulicas actuales y los factores que influyen en la reducción de la capacidad hidráulica, los que se presentan en la conducción superior, resultados que permiten establecer que las pérdidas se dan por un mínimo mantenimiento en las estructuras y válvulas, pérdida de carga local en la torre de captación del embalse, posible acumulación de sedimentos, y una posible acumulación de aire en el sistema. Para establecer alternativas de solución se modelaron cinco escenarios que simularon condiciones críticas, óptimas y medias de operación del embalse para diferentes caudales transportados, con lo que se determinaron presiones negativas que impiden el transporte del caudal máximo de diseño. De su análisis se concluyó que las alternativas de solución más viables para que a través de la tubería de conducción se transporte $1750 \mathrm{l} / \mathrm{s}$ son: el mantenimiento del sistema complementado con la modificación del perfil de la línea de conducción; o la implementación de una booster en la abscisa 2+908 de la conducción superior, con altura de bombeo de $30 \mathrm{mca}$.

Palabras clave: sistema La Mica - Quito Sur, pérdidas de energía, capacidad hidráulica, modelación hidráulica, presiones negativas.

\begin{abstract}
"La Mica - Quito Sur" System provides water to the southern area of Quito, it is currently carrying a maximum flow of $1560 \mathrm{l} / \mathrm{s}$, less than $1750 \mathrm{l} / \mathrm{s}$ for which it was designed, this decrease is due to factors that generate energy losses in the line of conduction which decreases its hydraulics capacity. We were carried out an intense activity to get an updated hydraulic model. Plans and system databases were collected and validated in situ. Then the hydraulic model was built, calibrated and validated, obtaining from its analysis the current hydraulic characteristics and the factors that influence the reduction of the hydraulic capacity, the same that are presented in the superior conduction, results that allow establishing that the losses are due to: the lack of maintenance in the structures and valves, loss of local load in the reservoir collection tower, possible accumulation of sediments and possible accumulation of air in the system. To establish alternative solutions, five scenarios were modeled to simulate critical, optimal and average conditions of operation of the reservoir for different transported flows, which determined negative pressures that prevents the transport of the maximum design flow. From their analysis was concluded that the most viable solution alternatives so that 1750 1/s can be transported through the pipeline are: maintenance of the system complemented by the modification of the profile of the line of conduction; or the implementation of a booster on the abscissa $2+908$ of the upper conduction, with pumping height of $30 \mathrm{~m}$ W.C.
\end{abstract}

Keywords: La Mica - Quito Sur system, energy losses, hydraulic capacity, hydraulic modeling, negative pressures. 


\section{I.Introducción}

De acuerdo con la Organización de Naciones Unidades (ONU) en la Asamblea General del derecho humano al agua y al saneamiento [1] "reconoció el derecho de todos los seres humanos a tener acceso a una cantidad de agua suficiente para el uso doméstico y personal, que sea segura, aceptable, asequible y accesible físicamente". De igual manera estipularon que "cada persona en la tierra requiere al menos 20 a 50 litros de agua potable limpia y segura al día para beber, cocinar o simplemente mantenerse limpios".

El incremento poblacional del Distrito Metropolitano de Quito genera un aumento de la demanda de agua potable, por lo tanto, se ve la necesidad de satisfacer esta demanda futura adicional para garantizar el derecho de toda la población de acceder al servicio de agua potable. De allí que es necesario mejorar las condiciones de funcionamiento de proyectos existentes de suministro de agua. La Empresa Pública Metropolitana de Agua Potable y Saneamiento (EPMAPS) en el Plan Maestro de Agua Potable [2] considera los principales proyectos de inversión a ejecutarse a corto y mediano plazo (2010-2019). Para La Mica - Quito Sur se proyecta un incremento de caudal de 180 l/s al caudal máximo de diseño de 1750 $1 / \mathrm{s}$, con lo cual se tiene una oferta de agua potable mayor a la demanda requerida por la población futura.

El sistema de agua potable La Mica - Quito Sur transporta agua cruda desde el embalse La Mica hasta la planta de tratamiento El Troje ubicada en la zona sur de Quito. El proyecto de evaluación hidráulica de la línea de conducción La Mica - Quito Sur, establece "condición crítica de caudal máximo de diseño $\mathrm{Q}=1750 \mathrm{l} / \mathrm{s}$ " [3], de igual manera este caudal se establece como: "capacidad hidráulica máxima real de $1750 \mathrm{l} / \mathrm{s}$ " [4]; debido a problemas operativos se ha llegado a determinar de manera experimental que el caudal máximo de operación es 1560 l/s.

Para aumentar el abastecimiento de agua cruda hasta la planta de tratamiento en 190 l/s, se debe analizar los factores que inciden en la disminución de la capacidad hidráulica de la línea de conducción, para alcanzar el caudal máximo de diseño de 1750 l/s. Es importante realizar la evaluación técnica del funcionamiento actual del sistema de conducción para poder abastecer a un mayor número de personas asentadas en la zona sur de Quito.

En este trabajo se realizó un análisis en flujo permanente de los factores que inciden en la disminución de la capacidad hidráulica de la línea de conducción del sistema La Mica - Quito Sur, para lograr este objetivo se examinó la información existente, como también se verificó el catastro del sistema con mediciones in situ, se construyó, calibró y validó un modelo hidráulico, finalmente se evaluó el funcionamiento del sistema de conducción bajo diferentes escenarios de funcionamiento y se recomendó posibles soluciones.

\section{Metodología}

\section{A. Ubicación}

El sistema La Mica - Quito Sur está ubicado en las provincias de Napo y Pichincha específicamente en el Distrito Metropolitano de Quito (DMQ), donde inicia con las fuentes de abastecimiento y termina en la planta de tratamiento El Troje respectivamente. Este sistema se encuentra divido en: conducción superior que va desde el embalse La Mica hasta la planta generadora de energía El Carmen y en la conducción inferior transporta agua desde El Carmen hasta la planta de tratamiento, como se indica en la figura 1.

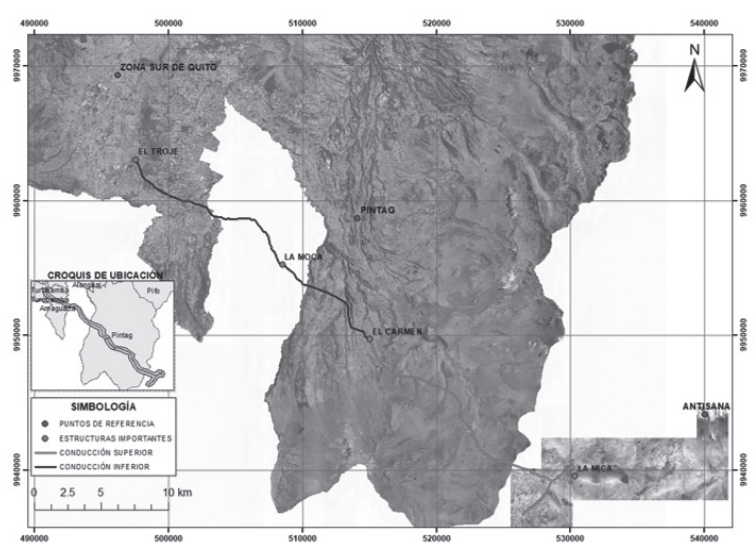

Fig. 1. Mapa de ubicación del sistema de conducción La MicaQuito Sur. 
El proyecto se encuentra a diferentes elevaciones, parte de los $3917 \mathrm{msnm}$ hasta los $3154.20 \mathrm{msnm}$ presentándose una variación de la temperatura en su trayecto donde: en el embalse La Mica se tiene temperaturas de 4 a $8^{\circ} \mathrm{C}$ y de 12 a $20^{\circ} \mathrm{C}$ en la planta de tratamiento.

\section{B. Población servida}

El sistema La Mica - Quito Sur dota de agua a la población asentada desde la Av. Morán Valverde hasta el límite cantonal sur de la ciudad, con una cobertura directa de $4000 \mathrm{Ha}$, de manera indirecta beneficia también a otros sectores de la ciudad como se puede muestra en la figura 2.

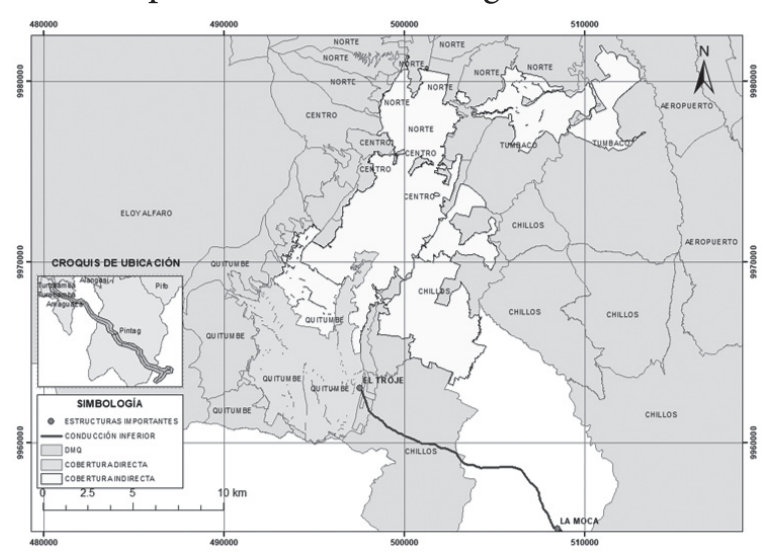

Fig. 2. Mapa de cobertura del sistema La Mica-Quito Sur.

En el año 2010 el sistema abastecía a 393.452 habitantes y a partir de este dato se realiza la proyección de la población a servir hasta el año 2040 observadas en la tabla 1.

Tabla 1 Proyección de la población servida

\begin{tabular}{|c|c|c|c|c|}
\hline Variables & 2010 & 2020 & 2030 & 2040 \\
\hline Población & 414160 & 482975 & 566999 & 656585 \\
\hline $\begin{array}{c}\text { Cobertura } \\
\text { sistema de agua }\end{array}$ & 99 & 99 & 99 & 99 \\
\hline $\begin{array}{c}\text { Población } \\
\text { servida }\end{array}$ & 393452 & 473315 & 555659 & 643453 \\
\hline $\begin{array}{c}\text { Dotación neta } \\
\text { 1/hab/día }\end{array}$ & 183 & 179 & 174 & 170 \\
\hline $\begin{array}{c}\text { Tasa de } \\
\text { crecimiento \% }\end{array}$ & 2.00 & 1.84 & 1.38 & 1.10 \\
\hline
\end{tabular}

El Plan Maestro de Agua Potable realizado por la EPMAPS [2] plantea el incremento de 180 l/s al caudal máximo de diseño de 1750 l/s, con este aumento se podrá abastecer a la población servida futura de 656.585 habitantes para el año 2040.

\section{Componentes del sistema}

Los componentes que conforman el sistema La Mica - Quito Sur se los puede clasificar en tres grupos principales: captaciones o fuentes de abastecimiento, líneas de conducción y obras complementarias, esta clasificación se visualiza en la figura 3.

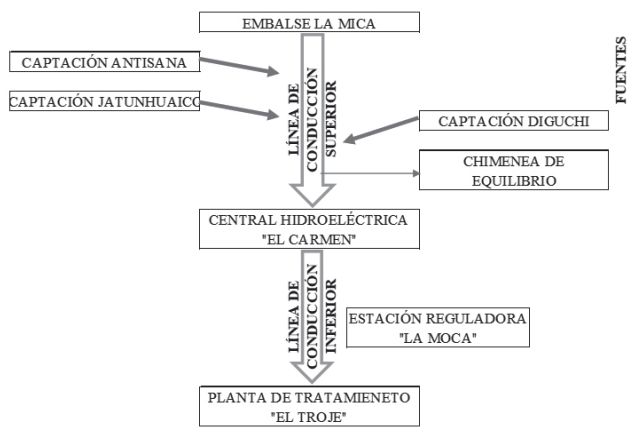

Fig. 3. Componentes del sistema La Mica - Quito Sur.

La línea de conducción superior empieza en el embalse y las captaciones hasta El Carmen donde se controla el caudal del sistema, la línea de conducción inferior comienza en el tanque de El Carmen hasta el tanque de El Troje y se regula el caudal desde La Moca.

\section{Construcción del modelo hidráulico}

En la construcción del modelo hidráulico se realizó el procedimiento de la figura 4. La actualización topológica es muy importante debido a que la calidad de la información ingresada en el modelo hidráulico tiene influencia directa con los resultados obtenidos. 


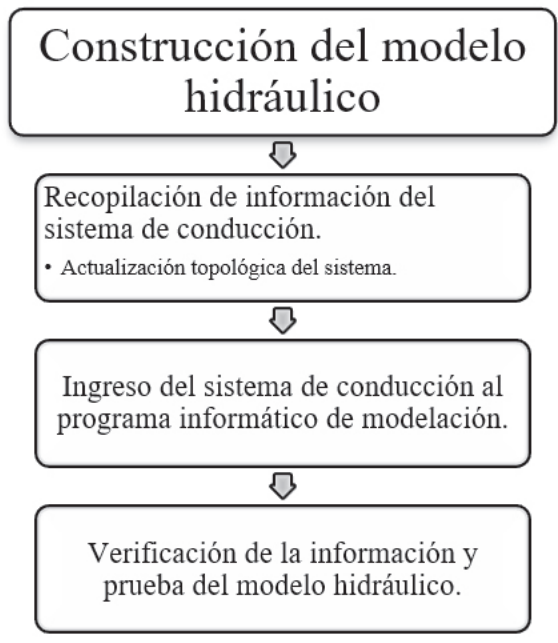

Fig. 4. Proceso de construcción del modelo hidráulico.

Se recopiló la siguiente información de la EPMAPS: planos as-built, fotos satelitales, estudios realizados anteriormente, topología existente del sistema, manuales y datos de las válvulas del sistema. Con la información existente se realizó una actualización topológica de los nodos y tuberías para realizar la modelación en EPANET como se muestra en la figura 5.

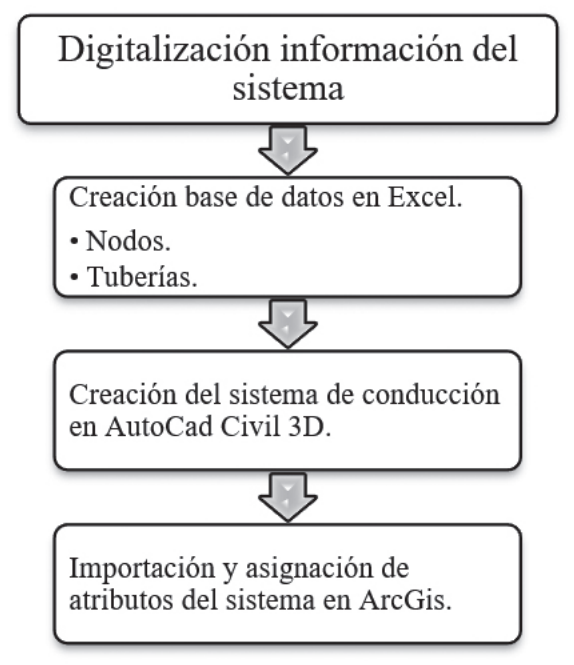

Fig. 5. Proceso de actualización topológica del sistema de conducción.

Para la topología se necesita la siguiente información de los nodos: nombre, abscisa, cota del terreno, cota de la tubería. De las tuberías: nodo inicial, nodo final, diámetro interno, espesor de la tubería, tipo y grado de material.

Posteriormente se ingresa la topología al programa informático EPANET para realizar una verificación de los datos ingresados como también un análisis de los resultados obtenidos en la primera modelación, estos resultados deben tener una diferencia de hasta $30 \%$ entre las mediciones y los resultados, para así poder realizar el proceso de calibración de acuerdo con lo planteado por la Asociación de Nueva Zelanda de Aguas y Desechos [5].

\section{E. Calibración del modelo hidráulico}

La calibración se realizó para la conducción superior e inferior de acuerdo con los pasos de la figura 6, donde se detalla el procedimiento para periodo estático y extendido.

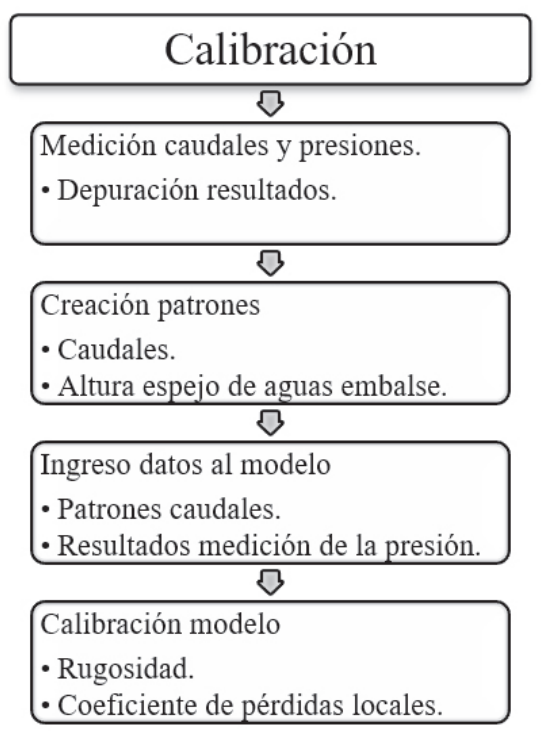

Fig. 6. Proceso de construcción del modelo hidráulico.

Los parámetros hidráulicos empleados en EPANET para las diferentes calibraciones realizadas se indican en la tabla 2, para la primera calibración se empleó un análisis estático, mientras que la segunda y tercera se modeló en periodo extendido. 
Tabla 2 Parámetros hidráulicos ingresados a EPANET.

\begin{tabular}{|l|r|}
\hline \multicolumn{1}{|c|}{ PARÁMETRO } & \multicolumn{1}{c|}{ DATO } \\
\hline Método de fricción & Darcy-Weisbach \\
\hline Tipo de análisis & Estático-Extendido \\
\hline Gravedad específica & 1 \\
\hline Temperatura del agua $\left({ }^{\circ} \mathrm{C}\right)$ & 12 \\
\hline Viscosidad cinemática $\left(\mathrm{m}^{2} / \mathrm{s}\right)$ & $1.24 * 10^{-6}$ \\
\hline
\end{tabular}

La instalación de los medidores de presiones o data loggers se realizó de acuerdo con la ubicación de los accesorios que generen pérdidas de carga considerable en el sistema, también en los puntos más críticos de la tubería, en la figura 7 se encuentra su ubicación para la conducción superior y en la figura 8 para la conducción inferior.

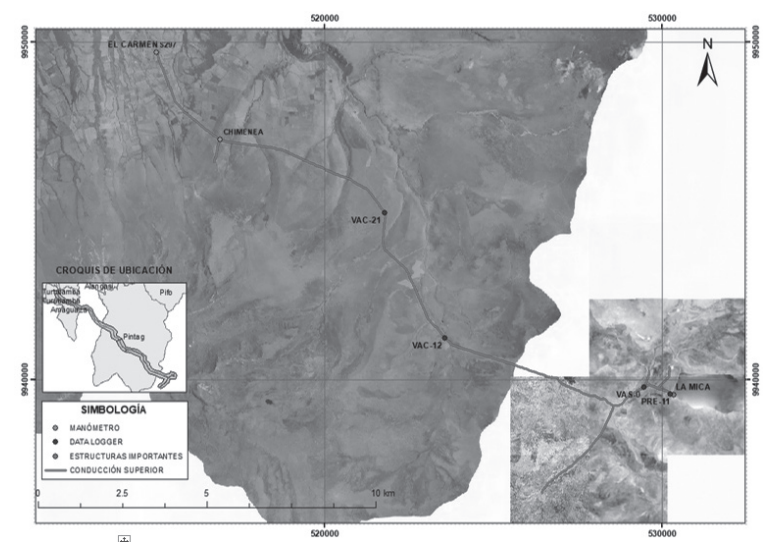

Fig. 7. Mapa de ubicación de manómetros y data loggers en conducción superior.

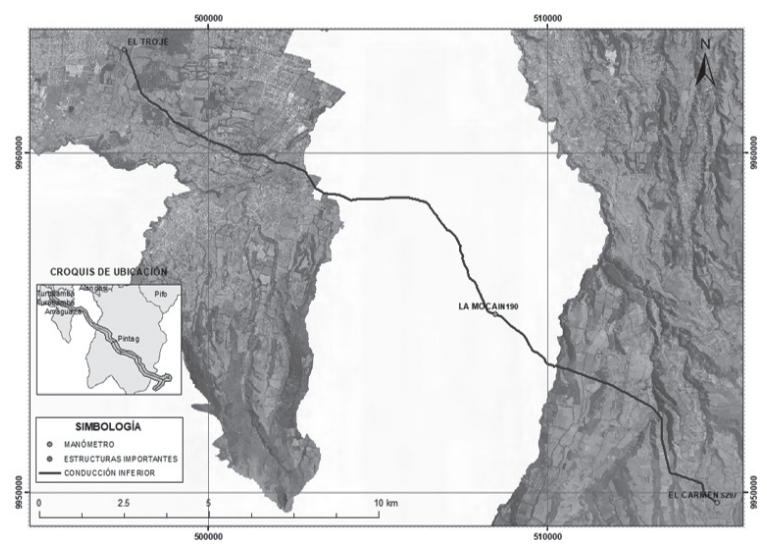

Fig. 8. Mapa de ubicación de manómetros en conducción inferior.
En la primera calibración para la conducción superior se emplean los datos proporcionados por el sistema SCADA de la EPMAPS donde los caudalímetros están ubicados en el embalse, las captaciones, a la entrada y salida de El Carmen; los manómetros están ubicados en la chimenea de equilibrio y en El Carmen (S297) como se observa en la figura 9.

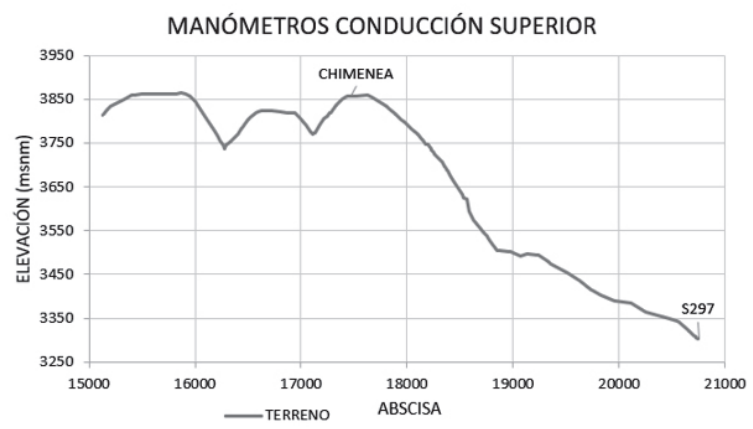

Fig. 9. Perfil de terreno entre abscisas $15+000$ a $21+000$ con manómetros en conducción superior primera calibración.

Se realizó un análisis para comprobar que los caudales estén dentro del primer criterio planteado por la Asociación de Autoridades del Agua (WAA) y El Centro de Investigación del Agua (WRC) [6] que se indica en la tabla 3, los caudales que cumplen este criterio son los modelados.

La rugosidad de diseño para tuberías de acero con revestimiento interno de coal-tar, enamel o epoxi es de $0.06 \mathrm{~mm}$ de acuerdo con la normativa vigente de la EPMAPS para el diseño de agua potable [7], dato inicial para la calibración del modelo por medio de un proceso de prueba y error. La calibración se realiza para dos tramos que van desde el embalse a la chimenea de equilibrio y desde la chimenea hasta El Carmen. Para las presiones se utilizaron los criterios de la tabla 3 . 
Tabla 3 Criterios prácticos para calibración de modelos según la WAA y WRC

\begin{tabular}{|c|}
\hline CRITERIOS I \\
\hline $\begin{array}{l}\text { 1) Precisión de } 5 \% \text { respecto a los caudales medi- } \\
\text { dos para caudales }>10 \% \text { de la demanda total; tu- } \\
\text { berías de conducción, generalmente de diámetro } \\
\text { mayor a } 16 \text { pulgadas }(406 \mathrm{~mm}) \text {. }\end{array}$ \\
\hline $\begin{array}{l}\text { 2) Precisión de } 10 \% \text { respecto a los caudales me- } \\
\text { didos para caudales }<10 \% \text { de la demanda total; } \\
\text { tuberías de distribución de diámetro generalmente } \\
\text { menor de } 12 \text { pulgadas }(305 \mathrm{~mm}) \text {. }\end{array}$ \\
\hline CRITERIOS DE PRESIÓN \\
\hline $\begin{array}{l}\text { 1) El } 85 \% \text { de las presiones medidas en pruebas } \\
\text { de campo no deben diferir en } 0.5 \mathrm{~m} \text { o } 5 \% \text { de la } \\
\text { pérdida de carga registrada. }\end{array}$ \\
\hline $\begin{array}{l}\text { 2) El } 95 \% \text { de las presiones medidas en pruebas } \\
\text { de campo no deben diferir } 0.75 \mathrm{~m} \text { o } 7.5 \% \text { de la } \\
\text { pérdida de carga registrada. }\end{array}$ \\
\hline $\begin{array}{l}\text { 3) El } 100 \% \text { de las presiones medidas en pruebas } \\
\text { de campo no deben diferir en } 2 \mathrm{~m} \text { o } 15 \% \text { de la pér- } \\
\text { dida de carga registrada. }\end{array}$ \\
\hline
\end{tabular}

Los resultados del primer día analizado se presentan a continuación en la tabla 4 , donde se tiene las diferentes iteraciones de la rugosidad para los tramos 1 y 2 con su respectivo error hasta cumplir lo planteado en la tabla 3 para la presión.

Tabla 4 Proceso de prueba y error para la primera calibración en conducción superior

\begin{tabular}{|c|c|c|c|c|c|c|c|c|c|}
\hline \multirow{3}{*}{$\begin{array}{c}\text { CORRI } \\
\text { DA }\end{array}$} & \multirow{3}{*}{$\begin{array}{l}\text { DATA } \\
\text { TIME }\end{array}$} & \multicolumn{2}{|c|}{ MEDIDOS } & \multicolumn{4}{|c|}{ SIMULADOS } & \multirow{2}{*}{\multicolumn{2}{|c|}{ Error P (\%) }} \\
\hline & & \multirow{2}{*}{\begin{tabular}{|c|}
$\begin{array}{c}\text { CHIMENE } \\
\text { A }\end{array}$ \\
$\begin{array}{c}\text { PIT201 } \\
\text { (mca) }\end{array}$ \\
\end{tabular}} & \multirow{2}{*}{$\begin{array}{c}\text { S297 } \\
\text { PIT301 } \\
\text { (mca) }\end{array}$} & \multirow{2}{*}{$\begin{array}{c}\begin{array}{c}\text { Tramo } 1 \\
\text { Rugosidad }\end{array} \\
(\mathrm{mm}) \\
\end{array}$} & \multirow{2}{*}{\begin{tabular}{|c|c}
$\begin{array}{c}\text { Tramo 2 } \\
\text { Rugosidad }\end{array}$ \\
$(\mathrm{mm})$ \\
\end{tabular}} & \multicolumn{2}{|c|}{ Presión (mca) } & & \\
\hline & & & & & & Chimene & S297 & Chimenea & S297 \\
\hline \multirow{4}{*}{$\frac{\frac{1}{2}}{3}$} & \multirow{4}{*}{ 1/11/2016 } & \multirow{4}{*}{23.537} & \multirow{4}{*}{570.000} & 0.060 & 0.060 & 30.480 & 574.090 & 22.778 & 0.712 \\
\hline & & & & 0.120 & 0.060 & 28.410 & 572.020 & 17.151 & 0.353 \\
\hline & & & & 0.210 & 0.060 & 26.050 & 572.020 & 9.646 & 0.353 \\
\hline & & & & 0.320 & 0.060 & 23.790 & 567.400 & 1.062 & 0.458 \\
\hline
\end{tabular}

Se obtuvo una rugosidad promedio de $0.829 \mathrm{~mm}$ para el tramo comprendido entre el embalse y la chimenea, en el tramo entre la chimenea y El Carmen se tiene una rugosidad de $0.06 \mathrm{~mm}$. El tramo 1 necesita una calibración más detallada debido al alto valor de rugosidad calculado.

Para la segunda calibración de la conducción superior se instalaron tres data loggers en los puntos más críticos de la conducción VAC21, VAC12 y en la válvula de control (VAS-0) como se mues- tra en la figura 7 y figura 10. Esta calibración se realiza en periodo extendido para garantizar que el modelo hidráulico se encuentre calibrado para los diferentes caudales de operación que se presentan en el sistema.

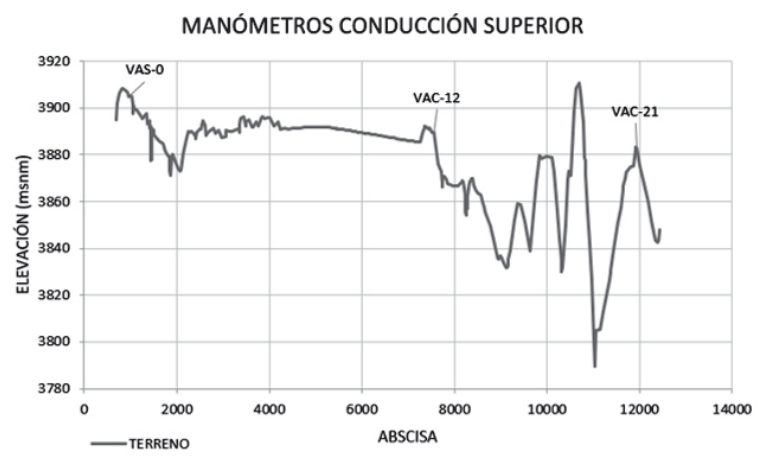

Fig. 10. Curva característica de la válvula de control (VAS-0), tipo mariposa.

Se realizó el procedimiento de la figura 6 y se busca cumplir lo planteado por la WAA y WRC en la tabla 3. Se ingresa también la curva característica de la válvula de control VAS-0 obtenida de la biblioteca del programa INFOWORKS, representada en la figura 11.

\section{CURVA VAS-0}

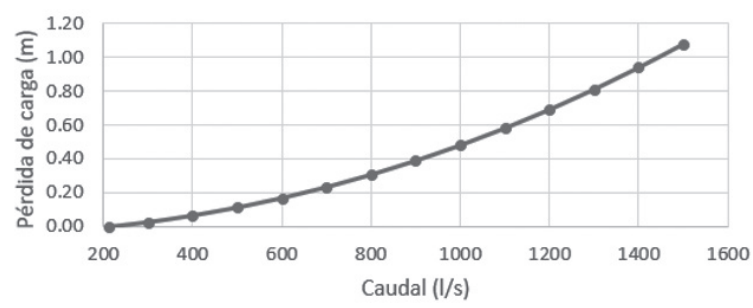

Fig. 11. Curva característica de la válvula de control (VAS-0), tipo mariposa.

Para el primer tramo comprendido entre el embalse y la VAS-0 se obtuvo una rugosidad igual $0.06 \mathrm{~mm}$ y un coeficiente de pérdida local en la válvula de control igual a 8 . Al analizar los datos de los resultados se obtuvo que el $96.01 \%$ de los resultados están dentro del $5 \%$ de la pérdida de carga registrada, el 99.96\% de los resultados están dentro del $7.5 \%$ de la pérdida de carga registrada y el $100.00 \%$ de los resultados están dentro del $15 \%$ de la pérdida de carga registrada, en la figura 
12 se observa la comparación entre las presiones medidas y calculadas.

PRESIONES VAS-0 (1406-B)
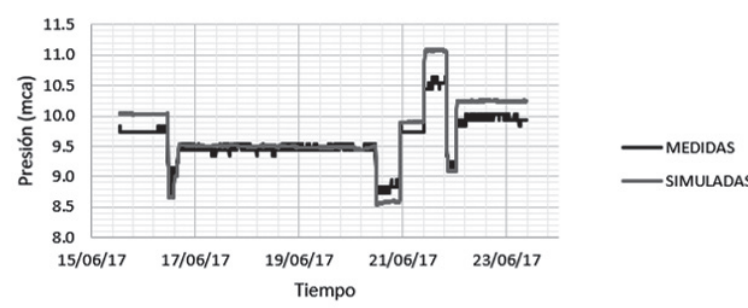

Fig. 12. Comparación presión VAS-0 (1406-B) simulada y medida en la segunda calibración conducción superior.

Para el resto de los tramos se realizó el mismo procedimiento y comparación para cumplir los criterios de calibración establecidos anteriormente en la tabla 3 , se tiene los siguientes resultados de la segunda calibración.

- Embalse - válvula de control $\mathrm{n}=0.6 \mathrm{~mm}$.

- Válvula de control - VAC-12 n=0.30 mm.

- VAC-12 - VAC-21 $\mathrm{n}=0.35 \mathrm{~mm}$.

- VAC-21 - chimenea $\mathrm{n}=0.9 \mathrm{~mm}$.

- Chimenea - El Carmen $\mathrm{n}=0.06 \mathrm{~mm}$.

- Válvula de control $\mathrm{k}=8$.

En el tramo del embalse y la válvula de control se encuentran accesorios que generan pérdidas de carga que necesitan un análisis más a detalle, razón por la cual se realiza la tercera calibración centrada en este tramo para determinar sus coeficientes.

En la tercera calibración se realizó el procedimiento detallado en la figura 6 y se utilizan los parámetros de calibración de la tabla 3 . Se instalaron los data loggers a la salida del túnel del embalse, antes y después de la válvula de control (VAS-0) como se muestra en la figura 7 y en la figura 13.

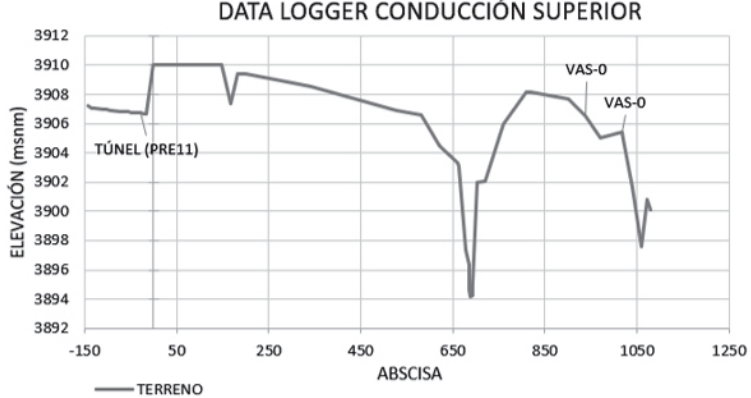

Fig. 13. Perfil de terreno con data loggers en conducción superior para la tercera calibración.

Para calibrar el tramo comprendido entre el embalse y el túnel del embalse o PRE-11 se colocó una rugosidad en la tubería igual a $0.2 \mathrm{~mm}$, se realizó una reducción de $0.485 \mathrm{~m}$ en el nivel del espejo de aguas del embalse. Al analizar los datos de los resultados se obtuvo que el $100 \%$ de los resultados están dentro del $5 \%$ de la pérdida de carga registrada, en la figura 14 se observa la comparación realizada de la presión medida y calculada.

PRESIONES PRE-11

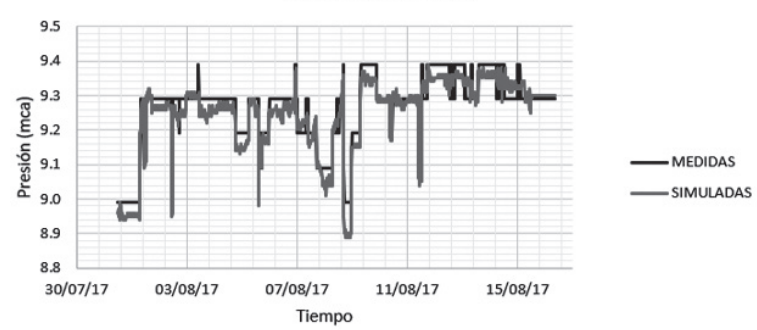

Fig. 14. Comparación presión PRE-11 simulada y medida.

Para el resto de los tramos se realizó el mismo procedimiento y comparación para cumplir los criterios de calibración establecidos anteriormente en la tabla 3 , se tiene los siguientes resultados de la tercera calibración.

- Embalse - PRE-11 n=0.2 mm.

- PRE-11 - válvula de control $\mathrm{n}=0.60 \mathrm{~mm}$.

- Válvula de control - chimenea $\mathrm{n}=0.6 \mathrm{~mm}$.

- Chimenea - El Carmen $\mathrm{n}=0.06 \mathrm{~mm}$.

- Tee conexión captación Antisana y Jatunhuayco a la conducción $\mathrm{k}=1.7$. 
- Válvula de control k=8.

- Pérdida de carga en la captación del embalse igual a $0.485 \mathrm{~m}$.

Para la calibración de la conducción inferior se utilizó los caudalímetros ubicados a la salida de El Carmen y entrada de El Troje y el manómetro instalado en La Moca de la figura 8 y figura 15, la primera calibración se realizó en periodo estático, mientras que la segunda en periodo extendido, las dos fueron realizadas de acuerdo el procedimiento de la figura 6 y cumpliendo los criterios de calibración de la tabla 3.

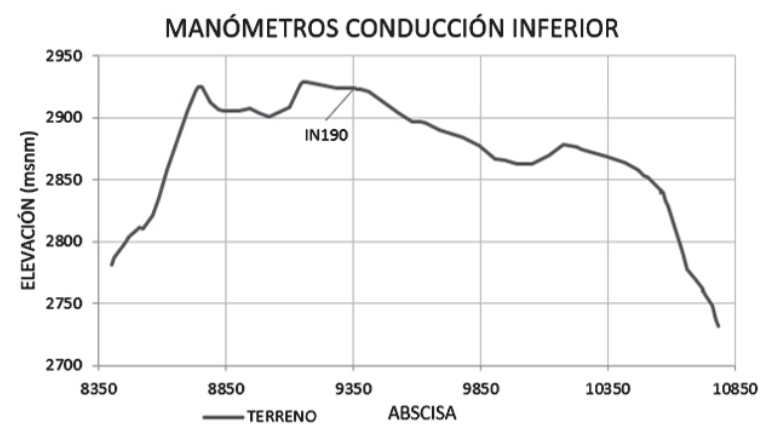

Fig. 15. Perfil de terreno entre abscisas $8+350$ a $10+850$ con manómetro en conducción inferior.

La rugosidad para la conducción inferior obtenido en la primera y segunda calibración es igual a $0.06 \mathrm{~mm}$ según la norma de diseño de agua potable de la EPMAPS [7], en esta línea no existe una pérdida de energía considerable de acuerdo con la rugosidad obtenida.

\section{Resultados y discusiones}

\section{A. Evaluación}

La evaluación del sistema se realizó para diferentes escenarios que fueron planteados de acuerdo con el caudal y la altura del espejo de agua del embalse.

Se tiene tres alturas diferentes que son: nivel máximo de operación de $3917.00 \mathrm{msnm}$, nivel medio de operación de $3913.38 \mathrm{msnm}$ y el nivel mínimo de operación de $3909.75 \mathrm{msnm}$. A estas cotas se realizó una corrección de $28.5 \mathrm{~cm}$ debido a la pérdida de carga que se genera al ingreso de la conducción en la torre de captación del embalse La Mica.

Los caudales que se modelaron son: condición actual de funcionamiento $1560 \mathrm{l} / \mathrm{s}$, caudal máximo de diseño 1750 l/s y caudal de 1930 l/s de acuerdo con el Plan Maestro de Agua Potable de la EPMAPS [2].

Se planteó los siguientes escenarios para representar condiciones óptimas, medias y críticas de funcionamiento en el sistema de conducción.

En la conducción superior:

- Escenario 1: Nivel máximo del espejo de agua (3916.715 msnm) con caudales aportados solo por el embalse (1560, 1750 y 1930 1/s).

- Escenario 2: Nivel medio del espejo de agua (3913.09 msnm) con caudales aportados solo por el embalse (1560, 1750 y 1930 1/s).

- Escenario 3: Nivel mínimo del espejo de agua (3909.465 msnm) con caudales aportados solo por el embalse (1560, 1750 y 1930 1/s).

- Escenario 4: Nivel medio del espejo de agua (3913.09 msnm) con caudales aportados solo por las captaciones (1560, 1750 y 1930 l/s).

En la conducción inferior:

- Escenario 5: Nivel mínimo del tanque en El Carmen (3301.15 msnm) con caudal de 1930 1/s.

La modelación del escenario 1 simula las condiciones más optimas de operación de la conducción superior cuando se encuentra únicamente funcionando el embalse. Con un caudal de 1560 1/s se observa que ya se presentan presiones negativas en el punto más crítico de la tubería el punto VAC-21 y al aumentar el caudal también aumentan las presiones negativas como se observa en la figura 16. 


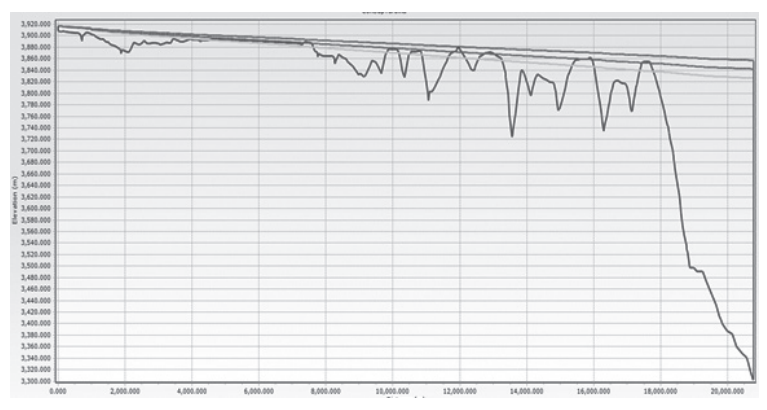

Fig. 16. Gráfica de comparación del gradiente hidráulico - perfil de la tubería del escenario 1 .

En los escenarios 2, 3 y 4 se presenta un mayor número de puntos con presiones negativas debido a que estos escenarios simulan condiciones más adversas que el primero.

El escenario 5 simula las condiciones más críticas de operación para la conducción inferior donde para un caudal de 1930 l/s no se presentan presiones negativas como se muestra en la figura 17.

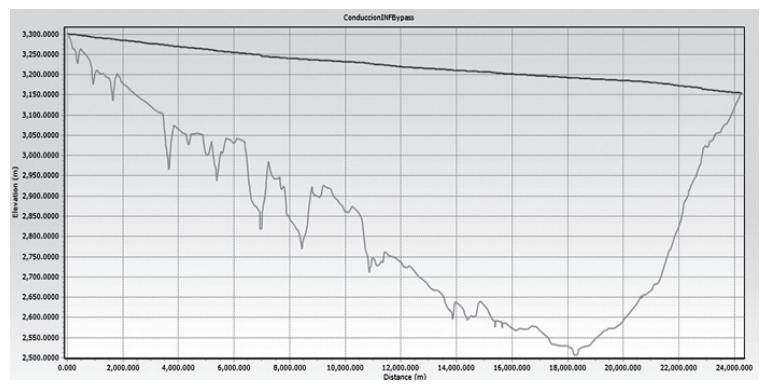

Fig. 17. Gráfica gradiente hidráulico - perfil de la tubería del escenario 5.

En la conducción superior se presentan presiones negativas para diferentes condiciones de operación como se verificó en la figura 16, por lo tanto, es importante plantear soluciones para evitar la generación de presiones negativas en la tubería y lograr transportar el caudal de 1930 1/s de cuerdo al Plan Maestro de agua potable de la EPMAPS [2].

La conducción inferior se encuentra en la capacidad de transportar $1930 \mathrm{l} / \mathrm{s}$ en sus condiciones actuales de operación debido a que no se presentan presiones negativas como se comprobó en la figura 17.

\section{B. Alternativas de solución}

Basándose en el análisis de los resultados de las calibraciones y escenarios, se establece que la conducción superior es la parte del sistema en la cual se encuentra la mayor pérdida de carga, por lo cual no permite el transporte máximo de los 1750 l/s, determinándose que las alternativas a formularse se aplican únicamente a esta parte del sistema.

La primera alternativa es la reducción de los coeficientes de rugosidad y pérdidas menores realizando un mantenimiento en las tuberías, accesorios, captaciones y válvulas para minimizar la pérdida de energía. Con esta alternativa se espera reducir la rugosidad en la tubería en todos los tramos aproximadamente a $0.06 \mathrm{~mm}$ y la pérdida de carga en la torre de captación debido al ingreso de la tubería igual a $0.285 \mathrm{~m}$.

Para modelar esta alternativa se redujo la rugosidad de las tuberías a $0.06 \mathrm{~mm}$, se consideró la altura más crítica del espejo de aguas sin corrección de 3909.75 msnm y un caudal de 1930 l/s. La figura 18 muestra los resultados obtenidos en la modelación donde se observan presiones negativas en los tramos indicados en la figura 19.

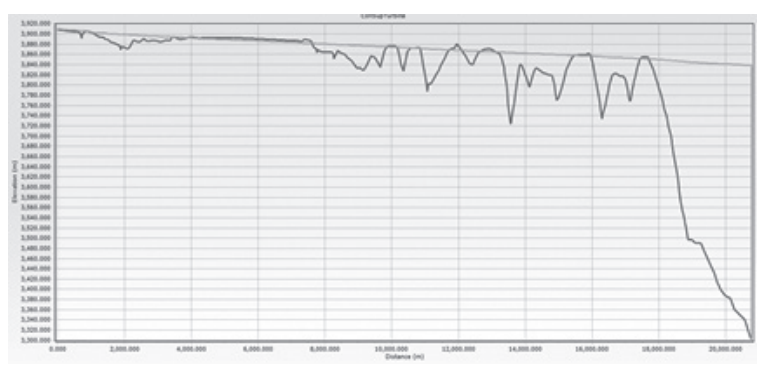

Fig. 18. Gráfica de comparación del gradiente hidráulico - perfil de la tubería, alternativa de reducción de los coeficientes de rugosidad y pérdidas menores.

Esta alternativa necesita ser complementada con un cambio del trazado de la tubería o la construcción de una línea paralela a la existente de menor diámetro que conduzca el caudal restante que no puede ser transportado en las condiciones actuales de operación, también se puede optar por la implementación de una booster. 


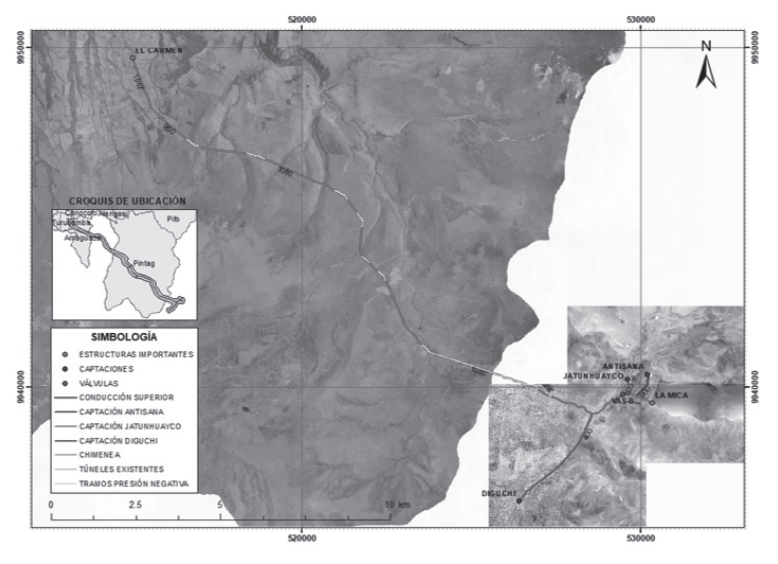

Fig. 19. Mapa de tramos con presión negativa de la alternativa 1.

Los tramos que necesitan un cambio de trazado debido a sus presiones negativas se muestran en la tabla 5, se presentan presiones negativas en una longitud total de $5482.58 \mathrm{~m}$.

Tabla 5 Tramos que requieren modificación de la línea de conducción superior

\begin{tabular}{|l|l|r|}
\hline \multicolumn{2}{|c|}{ Tramo crítico } & \multicolumn{1}{c|}{ Longitud } \\
\hline Nodo inicial & Nodo final & (m) \\
\hline VAC8 & S50 & 20.00 \\
\hline PI19 & S60.1 & 290.55 \\
\hline S61 & PI23 & 3309.78 \\
\hline VAC17 & PI34A & 262.47 \\
\hline POT20 & S165 & 184.29 \\
\hline VAC20A & S184 & 298.68 \\
\hline POT29 & PI40 & 359.10 \\
\hline PI54 & S237 & 500.36 \\
\hline POT53 & POT56 & 257.35 \\
\hline & TOTAL & 5482.58 \\
\hline
\end{tabular}

Para evitar la interrupción del sistema al construir el cambio de trazado de la línea de conducción, se modeló una booster ubicada después de las captaciones para mejorar su operación. La implementación de una booster tiene una inversión inicial menor al cambio de trazado o la construcción de una tubería paralela de menor diámetro, pero se debe considerar sus costos de operación a largo plazo.
La booster se instaló en el nodo S34 donde se encuentra la intersección entre la captación Diguchi y la línea de conducción, ubicada en la abscisa $2+098$ para garantizar el bombeo de agua del embalse y las captaciones como también una presión mínima de tres metros luego de su instalación de acuerdo con la figura 20.

Fig. 20. Mapa de ubicación de la booster.

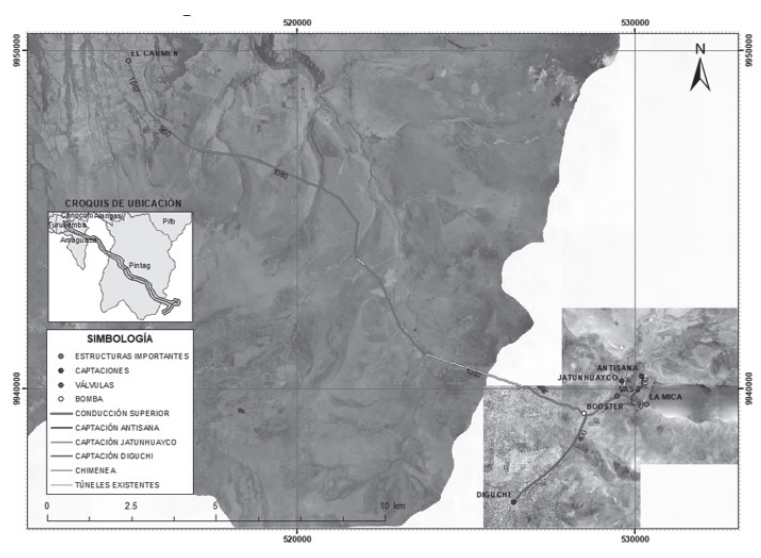

La ecuación 1 es de la bomba instalada y la figura 21 muestra su curva característica.

$$
\mathrm{H}=40-2.5 * 10^{-6} * Q^{2}
$$

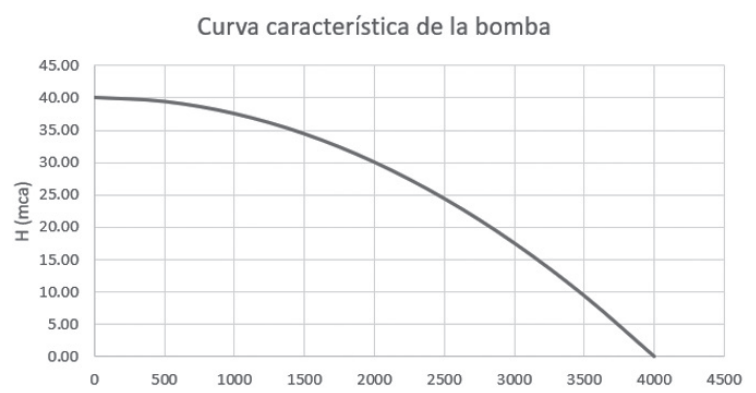

Fig. 21. Curva característica de la bomba instalada.

Para un caudal de 1930 1/s y una altura del espejo de agua del embalse de $3909.465 \mathrm{msnm}$ se obtuvieron las siguientes presiones: en el punto más crítico VAC-21 de 3.76 mca y en la chimenea de equilibrio de $7.57 \mathrm{mca}$. En la figura 22 se observa que el perfil longitudinal de la tubería y su gradiente hidráulico no se cortan, por lo tanto, no se presentan presiones negativas en esta modelación. 


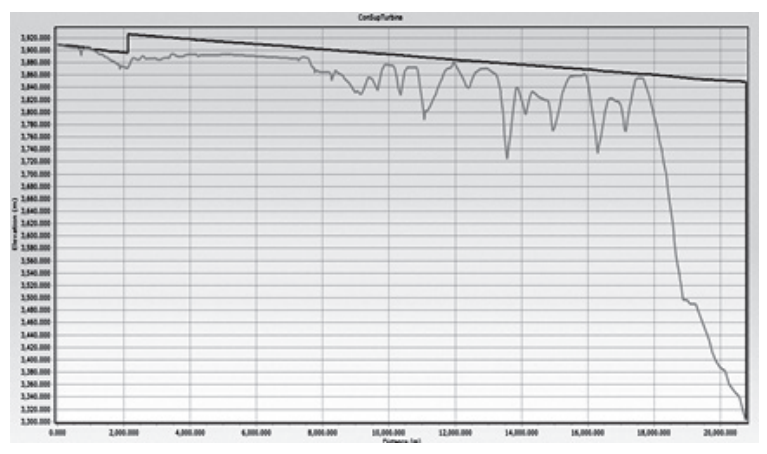

Fig. 22. Gráfica de comparación del gradiente hidráulico - perfil de la tubería, alternativa de implementación de una booster en la línea de conducción superior con un caudal de 1930 1/s.

La booster se enciende a los $1300 \mathrm{l} / \mathrm{s}$ y trabaja hasta los 1930 l/s, garantizando antes de su operación una presión mínima de 3 mca, para los 1300 1/s se tiene una presión de 3.04 mca en la VAC-21 y 19.18 mca en la chimenea de equilibrio respectivamente. La figura 23 muestra que no se presentan presiones negativas en este caudal.

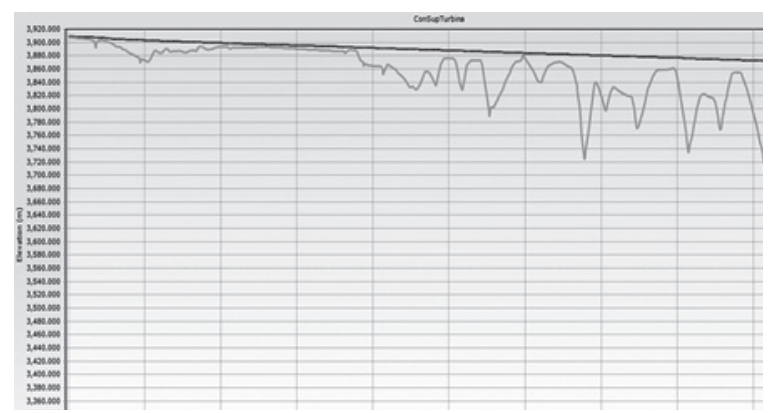

Fig. 23. Gráfica de comparación del gradiente hidráulico - perfil de la tubería, alternativa de implementación de una booster en la línea de conducción superior con un caudal de 1300 l/s.

Complementando esta alternativa con el mantenimiento de la línea de conducción se obtendrían mejores resultados teniendo mayores valores de presión en todos los nodos, pero se debe considerar cuáles serían las condiciones más críticas que se pueden presentar en el funcionamiento del sistema.

\section{Conclusiones y recomendaciones}

\section{A. Conclusiones}

En la torre de captación del embalse "La Mica" existe una pérdida de energía igual a $48.50 \mathrm{~cm}$ de acuerdo con la tercera calibración, donde 20.00 $\mathrm{cm}$ corresponden a la diferencia en la calibración del nivel máximo del embalse que es igual a $3917.00 \mathrm{msnm}$ según los planos de diseño definitivo, los $28.50 \mathrm{~cm}$ restantes se deben a la pérdida de energía local en la misma.

El tramo comprendido entre la salida del túnel del embalse (PRE-11, abscisa 0+113.95) y la válvula de control (VAS-0) ubicada en la abscisa 0+970, tiene una alta rugosidad de $0.60 \mathrm{~mm}$ presumiblemente por la gran cantidad de sedimentos arrastrados que provienen de la captación Antisana, por sus características geológicas y morfológicas.

Con la instalación de los data loggers utilizados para las calibraciones en la línea de conducción superior se determinó que existe una acumulación de aire en la válvula de aire VAC-12, influyendo negativamente en la capacidad hidráulica de la tubería y generando pérdidas de carga.

El tramo comprendido entre la válvula de control (VAS-0), ubicada en la abscisa 0+970 y la chimenea se encuentra operando con una rugosidad igual a $0.60 \mathrm{~mm}$, se asume que esta rugosidad no es la que existe en la tubería e indica la existencia de otros factores no considerados en la modelación en flujo permanente, en este tramo se encuentra la válvula de aire VAC-12 ubicada en la abscisa $7+556.26$ donde se puede considerar que la pérdida de carga no se debe únicamente a la rugosidad sino también al aire presente en el flujo que genera una contracción de la vena del fluido.

Los factores que influencian en la reducción del caudal máximo de diseño (1750 l/s) en el sistema La Mica - Quito Sur se encuentran presentes en la conducción superior, siendo los siguientes: mínimo mantenimiento en las estructuras, válvulas y medidores instalados, pérdida de carga elevada en la torre de captación del embalse siendo equivalente a una obstrucción del $50 \%$ de su sección, posiblemente una gran cantidad de sedimentos en el tramo comprendido entre PRE-11 y VAS-0, 
funcionamiento incorrecto de las válvulas de aire generando una disminución en la vena del fluido transportado.

La conducción superior con un caudal de 1560 1/s y una altura del espejo de aguas en el embalse igual a $3916.715 \mathrm{msnm}$, siendo el escenario más favorable de operación presenta problemas de funcionamiento generando presiones negativas en el nodo VAC-21 ubicado en la abscisa $11+922.67$.

Las características actuales de funcionamiento de la conducción inferior demuestran que en condiciones favorables permite el transporte del máximo caudal actual de 1560 l/s con el mínimo nivel del tanque de la central hidroeléctrica "El Carmen", siendo estas mismas condiciones aptas para transportar un caudal máximo de $1930 \mathrm{l} / \mathrm{s}$ con un nivel mínimo del tanque $3301.15 \mathrm{msnm}$ La reducción de los coeficientes de rugosidad y pérdidas menores en la línea de conducción superior sigue presentando presiones negativas en una longitud total de $5482.58 \mathrm{~m}$ donde se recomienda realizar un estudio a profundidad para realizar un cambio de trazado de la tubería en los respectivos tramos, siendo necesario complementar esta alternativa con la ejecución de otras soluciones.

La solución más viable para aumentar la capacidad hidráulica de la línea de conducción superior a 1930 l/s es la instalación de una booster en el nodo PI-1 ubicado en la abscisa $2+098$, donde la altura de bombeo es 30 mca para un caudal de diseño igual a $2000 \mathrm{l} / \mathrm{s}$, garantizando de esta manera una presión mínima de $3 \mathrm{~m}$ en todos los puntos aguas abajo de su instalación y operando entre los caudales de 1930 l/s y 1300 l/s.

\section{B. Recomendaciones}

Realizar mantenimiento periódico de todos los componentes del sistema, con especial enfoque a disminuir la rugosidad de las tuberías y revisar el correcto funcionamiento de las válvulas de control, desagüe y de aire, garantizando su adecuado funcionamiento con la reducción de las pérdidas de carga.

Mantenimiento, calibración y encerado de los equipos de medición que emiten información al sistema SCADA, como también de los equipos utilizados en los levantamientos topográficos y medición de presiones para los trabajos de campo, su reemplazo inmediato en caso de ser necesario y la instalación de un medidor de presiones en la válvula de aire VAC-21 permitirá operar la línea de conducción con mayor seguridad.

Modelar el sistema de conducción La Mica - Quito Sur en flujo no permanente luego de haber sido aplicadas las recomendaciones anteriores para garantizar la exactitud de los resultados y minimizar la inversión necesaria para la implementación de las soluciones.

\section{Agradecimientos}

Este trabajo fue posible gracias al apoyo de la Empresa Pública Metropolitana de Agua Potable y Saneamiento (EPMAPS) por proporcionar los datos, logística y personal de campo.

\section{Referencias}

[1] Naciones Unidas ONU. (2010). El derecho humano al agua y saneamiento. Naciones Unidas. Obtenido de: http: http://www. un.org/ga/search/view_doc.asp? symbol=A/ RES/64/292\&Lang=S

[2] EPMAPS. (2011). Resumen Ejecutivo Plan Maestro de Agua Potable. Quito.

[3] EPMAPS. (2006a). Fase I Diagnóstico y formulación de alternativas Volumen No 1. Quito.

[4] EPMAPS. (2006b). Fase I Diagnóstico y formulación de alternativas, resumen ejecutivo. Quito.

[5] The New Zealand Water and Wastes Association. (2009, April). National Modelling Guidelines Water Distribution Network Modelling. Modelling Special Interest Group.

[6] Water Authorities Association y Water Research Center. (1989). Network Analysis: A Code of Practice. Water Research Center.

[7] EPMAAP-Q. (2008). Normas de diseño de sistemas de agua potable para la EMAAP-Q. Quito: Empresa Metropolitana de Alcantarillado y Agua Potable. 